\title{
High blood pressure and low awareness on the preoperative evaluation
}

Hülya KAŞIKARA ${ }^{1}$, Dilek ÖZTAŞ², Şemsi M. AKSOY³

${ }^{1}$ Anesthesiology and Reanimation Clinic, Ankara Atatürk Training and Research Hospital, Ankara, Türkiye.

${ }^{2}$ Department of Public Health, Faculty of Medicine, Yıldırım Beyazıt University, Ankara, Türkiye.

${ }^{3}$ Department of Anesthesiology and Reanimation, Faculty of Medicine, Yıldırım Beyazıt University, Ankara, Türkiye.

\section{SUMMARY}

The most important risk factor for morbidity and mortality among patients with cardiovascular diseases undergoing surgery under anesthesia is hypertension (HT). Objective: This study aimed to investigate the prevalence and awareness of HT during preoperative evaluation. It was a descriptive and cross-sectional study. A total of 392 patients aged more than 30 years were included in the preoperative evaluation of the study. Results: The blood pressure levels of the patients were $8.1 \%$ optimum, $10.1 \%$ normal, $14.9 \%$ higher normal, $15.4 \%$ Stage 1 HT, $15.9 \%$ Stage 2 HT, $15.9 \%$ Stage 3 HT, and $18.7 \%$ isolated systolic HT. It was found that $63.6 \%$ of the 261 patients with high blood pressure were not diagnosed with HT and did not use any antihypertensive drugs. Hence, it was accepted that these patients were not aware of HT. Further, it was found that $42 \%$ of the patients were diagnosed with HT and received antihypertensive treatment. It was assumed that these patients were aware of HT.

Conclusions: Optimal clinical evaluation in the preoperative period in patients requiring surgical intervention may contribute to the reduction of perioperative morbidity through early diagnosis and treatment of HT and end-organ damage.

Key words: Awareness of hypertension, preoperative evaluation, preoperative risk

\section{INTRODUCTION}

Hypertension (HT) is a disease that affects more than one billion people worldwide and causes more than 7 million deaths per year (1). It is the most important risk factor for cognitive decline and premature death as well as many diseases that cause permanent disability (2). HT is the most important risk factor for morbidity and mortality among patients with cardiovascular diseases undergoing surgery under anesthesia. It is reported to be the most common comorbid disease in the elderly patient group. It is also the medical problem that causes maximum postponement of the operation $(3,4)$.

This study was performed to evaluate the frequency of HT and the level of awareness in patients undergoing preoperative anesthesia.

\section{MATERIALS AND METHODS}

This study was a descriptive cross-sectional study conducted in the anesthesia clinic of Ankara Atatürk Training and Research Hospital in March 2016. The Social and Human Sciences Ethics Committee of Ankara Yildirim Beyazit University approved for the study.

The inclusion criteria for patients were an age more than 30 years and an informed consent form from the patient. The exclusion criteria for patients were an age less than 30 years and the lack of informed consent form from the patient.

The 392 patients who met these criteria were included in the study. The gender and age of the patients who applied to the outpatient clinic were determined and their anamnesis was taken. Details including which surgery to undergo, previous 
TABLE 1: Definitions and classification of blood pressure $(\mathrm{mm} \mathrm{Hg})$ levels.

\begin{tabular}{llll}
\hline Category & Systolic & & Diastolic \\
\hline Optimum & $<120$ & and & $<80$ \\
Normal & $120-129$ & and/or & $80-84$ \\
High normal & $130-139$ & and/or & $85-89$ \\
Stage 1 hypertension (Stage 1 HT) & $140-159$ & and/or & $90-99$ \\
Stage 2 hypertension (Stage 2 HT) & $160-179$ & and/or & $100-109$ \\
Stage 3 hypertension (Stage 3 HT) & $\geq 180$ & and/or & $\geq 110$ \\
Isolated systolic HT & $\geq 140$ & and & $<90$ \\
Patient with diabetes & $\geq 140$ & and/or & $\geq 85$ \\
\hline
\end{tabular}

anesthesia history, disease history, medications, and cigarette use were recorded. Physical examinations were performed, during which pulse and blood pressure levels were measured and recorded. The patients were rested for 10 min before the pulse, and blood pressure were measured. Blood pressure measurement was done using the standard method. The patients were not allowed to take cigarettes and caffeine 30 min before measurements. A standard sleeve with a length of $35 \mathrm{~cm}$ and a width of $12 \mathrm{~cm}$ was used. For obesity and weakness, larger and smaller cuffs were available. The cuff was kept at the heart level. At least two measurements were made with a 10-min interval in the sitting position. More measurements were made in the case of a significant difference between the two measurements. The measurements were made in both arms, and high values were taken as reference. The evaluation was made according to Table 1 (5).

\section{Statistical analysis}

Statistical analyses were performed using the IBM SPSS (Statistical Package for the Social) for Windows Version 22.0 software package program. Numerical variables were summarized with mean \pm standard deviation. Categorical variables were expressed as number and percentage. A significance difference in categorical variables between the groups was investigated using the chi-square test. The normality of numerical variables was examined using Kolmogorov-Smirnov test. Differences between independent groups in terms of numerical variables were investigated using one-way analysis of variance. The significance level was considered as $P<0.05$.
Relationships between HT variable and gender, age, BMI (B history of diabetes and use of antihypertensive drugs were compared.

\section{RESULTS}

Of the 392 patients surveyed, $56.1 \%$ (220 patients) were males and $43.9 \%$ (172 patients) were females. Among surveyed patients, $49.9 \%$ were aged more than 60 years, $41.2 \%$ were in the $40-59$ age group, and $8.8 \%$ were in the $30-39$ age group. The average age of the patients was $58.4 \pm 12.7$. The minimum to maximum age range was 30-88. Among the patients, 30.6\% (120) said that they used blood pressure medication for their HT (Table 2).

\section{Blood pressure}

Among the patients, the blood pressure values of $8.1 \%$ (32 patients), 10.1\% (61 patients), and 14.9\% (59 patients) were optimum, normal, and high normal, respectively. Further, 15.4\% (61 patients) were at Stage 1HT, 15.9\% (63 patients) were at Stage 2 HT, 15.9\% (63 patients) were at Stage 3 HT, and 18.7\% (73 patients) had isolated systolic HT. Also, 63.6\% of them (261 patients) had high blood pressure and 33.4\% (131 patients) were bloodless normal (Table 3).

\section{HT/Gender}

Among the patients with high blood pressure, 42.1\% (110 people) were females and $57.9 \%$ (151 people) were males. No statistically significant correlation was found between HT level and gender $(P=0,263)$. 
TABLE 2: Demographic data.

\begin{tabular}{|c|c|c|c|}
\hline & & $n$ & $\%$ \\
\hline \multirow[t]{2}{*}{ Gender } & Female & 172 & 43.9 \\
\hline & Male & 220 & 56.1 \\
\hline \multirow[t]{3}{*}{ Age (year) } & $30-39$ & 35 & 8.8 \\
\hline & $40-59$ & 163 & 41. 2 \\
\hline & $\begin{array}{l}\text { More than } 60 \\
\text { years }\end{array}$ & 198 & 50.0 \\
\hline \multirow[t]{4}{*}{ BMI } & $\begin{array}{l}\text { 18, 50-24, } 9 \\
\text { (normal) }\end{array}$ & 99 & 28.9 \\
\hline & $\begin{array}{c}\text { 25-29, } 99 \\
\text { (overweight) }\end{array}$ & 133 & 38.8 \\
\hline & $\begin{array}{l}\text { 30-39, } 99 \\
\text { (obese) }\end{array}$ & 108 & 31.5 \\
\hline & $\begin{array}{l}>40 \\
\text { (morbid obese) }\end{array}$ & 3 & 0.7 \\
\hline \multirow[t]{6}{*}{ ASA } & 1 & 138 & 35.1 \\
\hline & 2 & 223 & 56.6 \\
\hline & 3 & 31 & 8.3 \\
\hline & 4 & & \\
\hline & $\mathrm{HT}$ & 120 & 30.6 \\
\hline & DM & 81 & 20.5 \\
\hline
\end{tabular}

Age: average $58.4 \pm 12.7$ minumum(min)-maximum(max); range 35-88. BMI: mean $=28.1 \pm 4.7 ;$ min- $\max =15.6-43.3 ; n=343$.

ASA: American Society of Anesthesiologists, DM: diabetes mellitus, HT: hypertension.

TABLE 3: HT classification.

\begin{tabular}{lcc}
\hline Level of HT & $N$ & $\%$ \\
\hline Normal & 131 & 36.4 \\
High & 261 & 63.6 \\
Total & 392 & 100.0 \\
\hline
\end{tabular}

\section{HT/Age}

Among the patients with high blood pressure, 59.4\% (155 people) were aged less than 65 years and 40.6\% (106 people) were aged more than 65 years. A statistically significant correlation was found between HT level and age ( $P=0.003)$.

Among the patients using blood pressure medication, 57.4\% (70 patients) were aged more than 65 years. Among the patients not using blood pressure medication, 25.5\% were aged more than 65 years. A statistically significant correlation was found between the use of blood pressure medication and age $(P<0.001)$.

\section{HT/Diabetes mellitus}

25.7\% (67 patients) of patients having high blood pressure were also diagnosed with diabetes mellitus (DM) A statistically significant correlation was found between HT level and DM $(P<0.001)$.

\section{HT/Antihypertensive drugs}

Among the 131 patients with normal blood pressure, 80.9\% (106 patients) did not use blood pressure medication and 19.1\% (25 patients) used blood pressure medication. Among the 261 patients with high blood pressure, 63.6\% (166 patients) did not use any blood pressure medication and 36.4\% (95 patients) used blood pressure medication. A statistically significant correlation was observed between high blood pressure and the use of blood pressure medication $(P<0.001)$ (Table 4).

Further, 27.0\% (106 patients) of 392 patients had normal blood pressure (A). Also, 63.6\% (166) did not use any antihypertensive drugs (C), and 36.4\% (95 people) used antihypertensive drugs (D) among 261 patients with high blood pressure $(C+D)$. In HT, awareness is defined as the detection and reporting of the presence of HT by a physician in hypertensive patients. It was accepted that

TABLE 4: Patients' HT levels and use of blood pressure pills.

\begin{tabular}{|c|c|c|c|c|c|c|c|}
\hline \multirow[b]{3}{*}{ HT level } & & \multicolumn{6}{|c|}{ Blood pressure medicine } \\
\hline & & \multicolumn{2}{|c|}{ Not taking medication } & \multicolumn{2}{|c|}{ Taking medication } & \multicolumn{2}{|c|}{ Total } \\
\hline & & $n$ & $\%$ & $N$ & $\%$ & $n$ & $\%$ \\
\hline & Normal & 106 & 80.9 & 25 & 19.1 & 131 & 100.0 \\
\hline & high & 166 & 63.6 & 95 & 36.4 & 261 & 100.0 \\
\hline & Total & 272 & 69.4 & 120 & 30.6 & 392 & 100.0 \\
\hline
\end{tabular}


TABLE 5: Detailed HT levels and medication use status of patients.

\begin{tabular}{|c|c|c|c|c|c|c|c|}
\hline \multirow[b]{3}{*}{ Blood pressure level } & & \multicolumn{6}{|c|}{ Blood pressure drug } \\
\hline & & \multicolumn{2}{|c|}{ Not taking medication } & \multicolumn{2}{|c|}{ Taking medication } & \multicolumn{2}{|c|}{ Total } \\
\hline & & $n$ & $\%$ & $n$ & $\%$ & $n$ & $\%$ \\
\hline & Optimum & 27 & 84.4 & 5 & 15.6 & 32 & 100.0 \\
\hline & Normal & 35 & 87.5 & 5 & 12.5 & 40 & 100.0 \\
\hline & High normal & 44 & 74.6 & 15 & 25.4 & 59 & 100.0 \\
\hline & Stage1 HT & 51 & 83.6 & 10 & 16.4 & 61 & 100.0 \\
\hline & Stage $2 \mathrm{HT}$ & 40 & 63.5 & 23 & 36.5 & 63 & 100.0 \\
\hline & Stage3 HT & 40 & 63.5 & 23 & 36.5 & 63 & 100.0 \\
\hline & Isolated systolic HT & 35 & 47.3 & 39 & 52.7 & 73 & 100.0 \\
\hline & Total & 272 & 69.4 & 120 & 30.6 & 392 & 100.0 \\
\hline
\end{tabular}

$P<0.001$. No difference was found between high-medication group and normal medication group; all other groups were different.

TABLE 6: Blood pressure status.

\begin{tabular}{ll} 
A & B \\
$(n=106)$ & $(n=25)$ \\
Normal blood pressure & Normal blood pressure \\
Not taking medication & $\begin{array}{l}\text { Taking medication } \\
\text { Awareness of hypertension } \\
\text { Drug effective }\end{array}$ \\
\hline C & D \\
$(n=166)$ & $(n=95)$ \\
High blood pressure & High blood pressure \\
Not taking medication & Taking medication \\
No awareness of hypertension & Awareness of hypertension \\
& Drug ineffective
\end{tabular}

people who did not use antihypertensive drugs in this group and had high blood pressure (C) did not have HT awareness. HT was diagnosed and 120 patients (42\%) were admitted with HT awareness $(B+D)$. It was seen that $20.8 \%$ (25 patients) of the 120 drug-using patients were aware and hence the drug was effective (B). However, the drug was ineffective in 79.2\% (D) (Table 6).

\section{DISCUSSION}

HT has a high prevalence worldwide, ranging from $20 \%$ to $50 \%$, according to World Health Organization data. It is also the leading cause of death in the world (6). Besides being the number one risk factor for preventable causes of death in the world, the perioperative rotation is also an important risk factor for prolonging cardiovascular problems (3). Hypertensive patients are more susceptible to hemodynamic instability and myocardial ischemia in the perioperative period (7). Various studies have shown a significant association between perioperative myocardial ischemia and postoperative ischemic cardiac events (unstable angina, myocardial infarction, and cardiac death) $(8,9)$.

Studies in different regions of the world have reported the prevalence of HT in general population as 45\%-63\%, awareness rate as $40 \%-88 \%$, treatment rate as $25 \%-84 \%$, and control rate as 20\%-65\% (10). A cross-sectional study by Palafox et al. in 2016 also investigated HT awareness, treatment, and control in 163,379 patients in 21 countries. In the present study, 42\% ( $B+$ D) patients had HT and antihypertensive drug use was reported as $41 \%$ (11). According to a comprehensive study conducted in 2005, the prevalence of HT was found to be $31.8 \%$, awareness rate $40.7 \%$, rate of treatment $31.1 \%$ and control rate $8.1 \%$ (12). In the present study, HT awareness was found and 19.1\% (120 patients) of 120 drug users were seen to have blood pressure control.

In a study conducted by Lwin et al., 26.3\% of patients aged 30-69 years who had HT were not aware of this condition beforehand (13). In the present study, 63.6\% had no awareness of HT. 
In a study conducted by Joffres et al in 2017, the prevalence, awareness, and treatment of HT between 2006 and 2010 in the United Kingdom (UK) ( $n=6873)$, the USA ( $n=10,003)$, and Canada ( $n=3485$ ) among patients with ischemic heart diseases was compared in the 20-79 age group. The prevalence of HT was found to be 30\% in the UK, 29\% in the USA, and 19.5\% in Canada. The prevalence of HT after 50 years of age was the lowest in the USA. HT awareness was close to $80 \%$ in the USA and Canada ( $81 \%$ and $80 \%$, respectively) and less in the UK (65\%). The levels of HT treatment were 51\% (UK), 74\% (USA), and 80\% (Canada) and the lowest among hypertensive patients (53\%) in Canada. The strongest correlation in this study was found between low awareness of HT and high stroke mortality (14). It was observed that 1070 (59.3\%) of the 1804 patients who detected HT in the HT prevalence study performed in Turkey were not aware of the high blood pressure (15). The present study found that 63.6\% (166 patients) of the 261 high-blood-pressure-hypertensive patients did not use any blood pressure medication and had no HT, leading to no HT awareness. It is stated that only one out of every three adults with HT in Turkey use antihypertensive medication due to the frequent and continuous follow-up of HT, side effects of medications, need to use several drugs, and inadequate education; only half of the patients taking medication can control blood pressure.

James et al. showed that the high blood pressure measured during preoperative evaluation was not sufficient to diagnose HT but should be investigated. Preoperative evaluation is a good opportunity to detect hypertensive disease and initiate appropriate treatment. It is important to determine the end-organ dysfunction caused by HT and detect HT in terms of predicting perioperative complications. Therefore, all patients with preoperative HT should be properly investigated using electrocardiogram, blood glucose analysis, electrolytes, and creatinine. It is considered a correct approach to delay elective surgery so as to investigate target organ damage and initiate treatment in patients with uncontrolled, Stage 3 HT (1). In the present study, urea creatinine values of patients with Stage 3 HT were assessed, and it was determined that 6 of 63 patients had renal dysfunction. Patients with blood pressure grade 3 HT and above were referred to the relevant departments for further investigation.
Studies have shown that an increase in systolic pressure by 20 $\mathrm{mm} \mathrm{Hg}$ or in diastolic pressure by $10 \mathrm{~mm} \mathrm{Hg}$ doubled the risk of developing cardiovascular diseases in patients aged $40-70$ years having a blood pressure 115/75 to 185/115 mm Hg. Therefore, the prevalence of HT, continuity of drug treatment, and complications such as myocardial ischemia, ventricular failure, impaired cerebral perfusion and peripheral vascular disease should be questioned before the anesthesia interview. The regular use of medications, exercise tolerance, shortness of breath, edema, postural hypotension, and syncope should be investigated (16).

HT is associated with hemodynamic instability in the preoperative period. Anesthesia induction and endotracheal intubation are hemodynamic instability periods for hypertensive patients (1). Regardless of preoperative blood pressure control levels, some patients with HT may show a markedly hypotensive response to anesthesia induction followed by an exaggerated hypertensive response to intubation. The systolic and diastolic pressures that develop immediately after the induction of anesthesia are much larger in hypertensive patients. Patients with a long-standing history of HT often have symptoms associated with ischemia, transmission anomalies, old infarction, or left ventricular hypertrophy (16). This leads to an increased risk of myocardial ischemia. Congestive heart failure is also an important risk factor for kidney and cerebral diseases, all of which increase perioperative risk (12). It is not certain whether HT without end-organ damage is a perioperative risk factor. Still it is not considered as a reason to cancel surgery (4).

White coat HT is closely related to anesthesia practice. This expression refers to a blood pressure greater than $140 / 90 \mathrm{~mm} \mathrm{Hg}$ in the outpatient clinic or preoperatively, despite blood pressures less than 135/85 mm Hg measured during normal daytime conditions. The patient with white coat $\mathrm{HT}$ is confused during the investigation and the decision to cancel the operation is complicated. Hence, the presence of any organ damage due to chronic HT should be evaluated at different times for several weeks for anesthesiologists when the mean blood pressure is not found. In the HT prevalence study conducted in Turkey, the blood pressure was found to be high when 59.3\% of the population was not HT-diagnosed (15). In the present study, 63.6\% had no HT awareness. Some of the 
patients having high blood pressure had "white coat HT." However, compared with the field study, no significant difference was found between them, and the number of patients detected with 15.9\% Stage 3 HT level was also quite meaningful.

Rapid control of blood pressure before surgery is not recommended in elective surgery patients, suggesting that the major organ systems may cause hypoperfusion during anesthesia. It is recommended to perform HT therapy in a stable manner within a sufficient time to reduce the perioperative risks of HT (18). The meta-analysis by Howell et al. suggested that perioperative outcomes were worse in hypertensive patients than in normotensive patients (7).

The number of patients with abortion due to high blood pressure at Stage $3 \mathrm{HT}$ after surgery is high in the operating room. These patients are usually not diagnosed or diagnosed with HT. Also, preoperative blood pressure is unregulated in the operating room, leading to loss of time, efforts, and costs in the operation. Taking this into consideration, it is suggested that patients who have applied to anesthesia clinic for preoperative evaluation should not skip blood pressure measurement within a careful history and good physical examination.

Stages I and II HT indicate no postponement of surgery for treatment optimization in patients without end-organ dysfunction and other risks (diabetes, kidney failure, and smoking) (7). However, in the present study, $25.7 \%$ of the patients with high blood pressure had type II diabetes at the same time, with a statistically significant correlation between blood pressure level and DM $(P<$ 0.001). At this point, preoperative blood pressure follow-up and antihypertensive treatment should Premedication preoperatively in appropriate patients may also help in operating these patients with a more stable blood pressure. In patients with Stage 3 HT (systolic blood pressure $>180 \mathrm{~mm} \mathrm{Hg}$ and/or diastolic blood pressure $>110$ $\mathrm{mm} \mathrm{Hg}$ ), the potential benefit and risk of postponing the operation to manage pharmacologic therapy should be well measured and decided. be conducted. It is recommended to initiate beta-blockers in the perioperative period of hypertensive disease, especially ischemic heart disease, which is associated with the target organ damage and cardiovascular problems recognized in patients with preoperative European Society of Hypertension (ESH) and treated according to the current and of the European Society of Cardiology
(ESC) guideline (2009). Antihypertensive therapy should continue until the morning of operation and should be started immediately postoperatively. It is considered that the most appropriate approach is to conduct necessary pre-operation clinical measurements and follow-ups and apply premedication in daily operations.

\section{Limitations}

Elevated values measured on at least two separate days in the diagnosis of HT and control programs imply HT. Because of fluctuations in blood pressure, the diagnosis of HT should be based on multiple blood pressure measurements made at different times. In the present study, HT was assessed with two measurements in one patient. Ambulatory blood pressure measurement after a blood pressure measurement of $140 / 90 \mathrm{~mm} \mathrm{Hg}$ or higher makes the diagnosis of HT more reliable.

This study aimed to determine the prevalence of uncontrolled HT, which is the most common medical problem encountered in the operating room, and the awareness of the patients. Optimal clinical evaluation in the preoperative period in patients requiring surgical intervention may contribute to the early recognition of HT and target organ damage and the reduction of perioperative morbidity, thus increasing the quality of patient care and the effective use of the operating theater. HT awareness studies are generally carried out as field studies. The number of patients was low and the follow-up time was short in the present study. Hence, largesample studies are needed to validate the findings.

\section{REFERENCES}

1. MFM James, RA Dyer, BL Rayner. A modern look at hypertension and anaesthesia, Journal of Endocrinology, Metabolism and Diabetes of South Africa, 2014 19:2, 56-6

2. Spahn DR. Preoperative hypertension: remain wary? "yes" cancel surgery? "No" Br J Anaesth 2004;92(4):461-464. 2

3. Howell SJ, Sear YM, Yeates D, Goldacre M, Sear JW, Foex P. Risk factors for cardiovascular death after elective surgery under general anesthesia. Br J Anaesth 1998; 80: 14-9.

4. Ahmet Selim Özkan. Hipertansiyon ve Hipertansif Hastalarda Anestezi. Derleme. İnönü Üniversitesi Sağlık Bilimleri Dergisi 2012; 2: 6-8.

5. Mancia G, De Backer G, Dominiczak A, et al. 2007 Guidelines for the management of arterial hypertension: The Task Force for the Management of Arterial Hypertension of the European Society of Hypertension (ESH) and of the European Society of Cardiology (ESC). Eur Heart J. Jun 2007;28(12):1462-1536. 
6. Lewington S, Clarke R, Qizilbash N, Peto R, Collins R. Age-specific relevance of usual blood pressure to vascular mortality: a metaanalysis of individual data for one million adults in 61 prospective studies. Lancet. Dec 14 2002;360(9349):1903-1913.

7. Howell SJ, Sear JW, Foëx P. Hypertension, hypertensive heart disease and perioperative cardiac risk. $\mathrm{Br} J$ Anaesth 2004;92(4):570-583

8. Mangano DT, Browner WS, Hollenberg M, London MJ, Tubau JF, Tateo IM. Association of perioperative myocardial ischemia with cardiac morbidity and mortality in men undergoing noncardiac surgery. The Study of Perioperative Ischemia Research Group. N Engl J Med 1990; 323: 1781-8

9. Landesberg $\mathrm{G}$, Luria MH, Cotev S, et al. Importance of longduration postoperative ST-segment depression in cardiac morbidity after vascular surgery. Lancet 1993; 341: 715-19

10. Wyatt SB, Akylbekova EL, Wofford MR, Coady SA, Walker ER, Andrew ME, Keahey WJ, Taylor HA, Jones DW:Prevalence, awareness, treatment, and control of hypertension in the Jackson Heart Study. Hypertension 2008;51:650-656.

11. Palafox B, McKee M, Balabanova D, AlHabib K. F, Avezum A. JR, Bahonar A, et al. Wealth and cardiovascular health: a crosssectional study of wealth-related inequalities in the awareness, treatment and control of hypertension in high-, middle- and lowincome countries, International Journal for Equity in Health 2016; 15:199

12. Arici $M$, Turgan $C$, Altun $B$, et al. Hypertension incidence in Turkey a population-based study. J Hypertens 2010;28(2):240244.5 .
13. Lwin M O, Malik S, Chua T S J, Chee T S, Tan Y S. Intergeneral transfer of blood pressure of blood pressure knowledge and screening: a school-based hypertension awareness program in Singapore, IUHPE- Global Health Promotion. 2016. Vol 23, No. 1, 2016 27-36. 1757-9759.

14. Joffres M., Falaschetti E, Gillespie C, Robitaille C, Loustalot F, Poulter N., McAlister F., Johansen H.,Baclic O.,Campbell N. Hypertension prevalence, awareness, treatment and Control in national surveys from England, the USA and Canada, and correlation with Strokes and ischaemic heart disease mortality : a cross-sectional study. British Med. J. 2013: e003423, doi:10.1136/ bmjopen-2013-003423

15. Mustafa Arıcı, Bülent Altun, Yunus Erdem, Ülver Derici, Gökhan Nergizoğlu, Çetin Turgan, Şükrü Sindel, Bülent Erbay, Oktay Karatan, Enver Hasanoğlu, Şali Çağlar, Türk Hipertansiyon ve Böbrek Hastalıkları Derneği. Türk Hipertansiyon Prevalans Çalışması (Paten T2). 2012

16. Morgan GE, Mikhail MS. Anaesthesia for Patients with Cardiovascular Disease. Clinical Anesthesiology, 4th Ed. Appleton \& Langepres. 2002; 389-95

17. Celis H, Staessen JA, Thijs L, Buntinx F, De Buyzere M, Den Hond $\mathrm{E}$, Fagard RH, O'Brien ET. Cardiovascular risk in white-coat and sustained hypertensive patients. Blood Pressure 2002;11:352356

18. Lines $D$, Anaesthetics Supplement: Hypertension and Anaesthesia. Department of Anaesthesia, Chris Hani Baragwanath Academic Hospital, 2014. University of Witwatersrand. 